\title{
ON THE FRATTINI SUBGROUPS OF GENERALIZED FREE PRODUCTS
}

\author{
BY R. B. J. T. ALLENBY AND C. Y. TANG ${ }^{1}$ \\ Communicated by George Seligman, July 17, 1973
}

1. Introduction. In [1] Higman and Neumann answered a question of $\mathrm{N}$. Ito whether free products of groups necessarily have maximal subgroups by showing that the Frattini subgroup of any free product of groups is trivial. From this it follows that free products of groups always have maximal subgroups. In the same paper Higman and Neumann raised the questions whether generalized free products of groups necessarily have maximal subgroups and whether the Frattini subgroup of a generalized free product is always contained in the amalgamated subgroup. No substantial result in the way of answering these questions had been obtained until Whittemore [5] showed that if $G=(A * B)_{H}$ is the generalized free product of $A$ and $B$ amalgamating $H$ and if there exists $x \in G$ such that $H \cap H^{x}=1$ then the Frattini subgroup $\Phi(G)$ of $G$ is trivial. Using this result Whittemore, in the same paper, showed that the Frattini subgroup of the generalized free products of finitely many free groups amalgamating a cyclic subgroup is trivial. This result was improved by Tang [4]: If $G=(A * B)_{H}$, where $A$ and $B$ are free and $H$ is finitely generated such that at least one of $[A: H]$ and $[B: H]$ is infinite then $\Phi(G)=1$. In the same paper it was also shown that if $G=(A * B)_{H}$ and $\Phi(G) \cap H=1$ then $\Phi(G)=1$. We can now greatly improve this result.

2. Main Theorem. Notations and terminology will be the same as in [4].

THEOREM 1. Let $G=(A * B)_{H}$. If $G$ contains a nontrivial normal subgroup $N$ such that $N \cap H=1$ then $\Phi(G)$ is contained in the maximal $G$ normal subgroup contained in $\mathrm{H}$.

\footnotetext{
AMS (MOS) subject classifications (1970). Primary 20E20, 20E30, 20F30; Secondary 20E25, 20F50.

Key words and phrases. Frattini subgroup, free groups, free products, generalized free product, amalgamated subgroup, permutational product, amalgam, $G$-normal, identical relation.

${ }^{1}$ The work of the second author was partly supported by a grant from the Canadian National Research Council No. A-4064.
} 
Proof. (Sketch). We can assume $H$ to be $G$-normal free. Thus $[A: H]$ and $[B: H]$ cannot both be 2 . Suppose $N \cap \Phi(G) \neq 1$. As in $[4$, p. 64] there exists $c \in N \cap \Phi(G)$ of the form $c=b_{1} a_{1} \cdots b_{k} a_{k}$ where $a_{i} \in A \backslash H$ and $b_{i} \in B \backslash H$. Clearly $c^{2} \neq 1$. Consider $X=\left\langle A^{c^{2}}, B\right\rangle$. If $c \in X$ then

$$
c=b_{0} c^{-2} a_{1} c^{2} b_{1} \cdots c^{-2} a_{n} c^{2} b_{n}
$$

where $n$ is chosen to be as small as possible and $b_{0}, b_{n}$ can possibly be 1 . If $c^{-1} a_{i} c \in H$ then $a_{i} \in H$ whence $\left[a_{i}, c\right] \in H \cap N=1$. In the same way $c b_{i} c^{-1} \in H$ implies $\left[b_{i}, c\right]=1$. Both cases are impossible because of the minimal choice of $n$. It follows that (1) cannot hold as the lengths of the two sides of (1) cannot be equal. Thus $c \notin X$ whence $c \notin \Phi(G)$. Therefore $N \cap \Phi(G)=1$ which implies $[N, \Phi(G)]=1$. But this is impossible unless $\Phi(G) \subseteq H$ since at least one of $[A: H]$ and $[B: H]$ is greater than 2 . Hence $\Phi(G)=1$. The theorem follows immediately.

Corollary 2. Let $\mathfrak{U}=(A, B ; H)$ be an amalgam amalgamating the subgroup $H$. If there exists an embedding of $\mathfrak{A}$ into a group $P=\langle A, B\rangle$ such that $A \cap B=H$ and $P$ is not isomorphic to $G=(A * B)_{H}$ then $\Phi(G)$ is contained in the maximal $G$-normal subgroup contained in $H$.

Since most amalgams amalgamating one subgroup have more than one embedding, Corollary 2 shows that a large class of generalized free products amalgamating one subgroup has maximal subgroups and more specifically their Frattini subgroups are contained in the amalgamated subgroups.

3. Some applications of Theorem 1. In [4] it was shown that if $G=$ $(A * B)_{H}$ where $A$ and $B$ are finitely generated torsion-free nilpotent groups and $H$ is closed in both $A$ and $B$ then $\Phi(G)$ is contained in the maximal $G$-normal subgroup contained in $H$. Applying Theorem 1 we can extend this result to the following:

TheOREM 3. Let $G=(A * B)_{H}$ where $A$ and $B$ are finitely generated nilpotent groups. Then $\Phi(G)$ is contained in the maximal G-normal subgroup contained in $H$.

Exploiting Theorem 1 in the direction as indicated by Corollary 2 and making use of permutational products as introduced by B. H. Neumann [2] we can prove the following results:

THEOREM 4. Let $G=(A * B)_{H}$ where $A$ and $B$ each satisfy a nontrivial identical relation and $H$ is infinite cyclic. If $A$ and $B$ each possess $a$ nontrivial abelian normal subgroup then $\Phi(G)$ is contained in the maximal $G$ normal subgroup contained in $H$. 
THeOREM 5. Let $G=(A * B)_{H}$. If $A$ and $B$ are locally solvable and $H$ is infinite cyclic then $\Phi(G)$ is contained in the maximal $G$-normal subgroup contained in $H$.

Applying Corollary 2 and making use of known results on embedding of amalgams as given by Corollary 6.5 and Theorems $6.6,8.4$ in [3] we obtain:

Theorem 6. Let $G=(A * B)_{H}$. If $A$ and $B$ have finite exponents and $H$ is central in one of them then $\Phi(G)$ is contained in the maximal G-normal subgroup contained in $H$.

THEOREM 7. Let $G=(A * B)_{H}$. If $A$ is periodic and $B$ is of finite exponent and $H$ is central in $A$ then $\Phi(G)$ is contained in the maximal $G$-normal subgroup contained in $H$.

THEOREM 8. Let $G=(A * B)_{H}$. If $A$ and $B$ are locally finite and $H$ is central and of countable index in one of them then $\Phi(G)$ is contained in the maximal G-normal subgroup contained in $H$.

The proofs of the above results will appear elsewhere.

REMARK. Although the results are stated in terms of generalized free products of two groups, they can be extended to generalized free products of more than two groups with a single amalgamated subgroup.

\section{REFERENCES}

1. G. Higman and B. H. Neumann, On two questions of Ito, J. London Math. Soc. 29 (1954), 84-88. MR 15, 286.

2. B. H. Neumann, Permutational products of groups, J. Austral. Math. Soc. 1 (1959/ 1960), 299-310. MR 23 \#A922.

3. - Amalgams of periodic groups, Proc. Roy. Soc. London Ser. A 255 (1960), 477-489. MR 22 \#4758.

4. C. Y. Tang, On the Frattini subgroups of certain generalized free products of groups, Proc. Amer. Math. Soc. 37 (1973), 63-68.

5. Alice Whittemore, On the Frattini subgroup, Trans. Amer. Math. Soc. 141 (1969), 323-333. MR 39 \#6993.

School of Mathematics, University of Leeds, Leeds, England

Department of Pure Mathematics, University of Waterloo, Waterloo, Ontario, CANADa 